\title{
Effects of Pulsed-radiofrequency Electrical Stimulation on the Rat with Detrusor overactivity
}

\author{
En Jen, Chih-Wei Peng, and Chii-Wann Lin
}

\begin{abstract}
The aim of this research was to develop a new electrical stimulation method for improving detrusor overactivity and verify its effect in animal experiments. In recent years most researchers use high frequency electrical stimulation at frequencies between $1 \mathrm{KHz}$ to $30 \mathrm{KHz}$, but the parameters and mechanism of this methods are not good enough to use on the clinical. This paper demonstrated the use of low-volt pulsed radio frequency electrical stimulation (frequency $500 \mathrm{KHz}$, pulse frequency $2 \mathrm{~Hz}$, pulse duration $80 \mathrm{msec}$, amplitude $\pm 3 \mathrm{~V}$ ) as a method to increase bladder capacity $(\mathrm{BC})$ by electrical stimulation on pelvic nerve. The primary results demonstrated that pulsed radio frequency electrical stimulation could inhibited acetic acid induced detrusor overactivity and significantly increased $\mathrm{BC}$ from $65 \%$ to approximately $102 \%-110 \%$, this modality can be used as an alternative approach for improving bladder continence in patients with detrusor overactivity.
\end{abstract}

Keywords-acetic acid, electrical stimulation, detrusor overactivity, pelvic nerve, pulsed radiofrequency.

\section{INTRODUCTION}

The lower urinary tract has two main functions: storage and voiding (i.e. periodic elimination of urine). Voiding depends on the integrated behaviors of the external urethral sphincter (EUS) and bladder, which is mainly dominated by lumbosacral spinal cord. EUS relaxes and bladder contracts during voiding, whereas the bladder relaxes and EUS contracts during storage [1]. Normally, pudendal and pelvic nerves directly exert neuromodulatory influences on EUS and bladder activity. The pudendal nerve (PN) is the primary conduit for somatic afferent and efferent nerve axons between the sacral spinal cord and urethra. PN plays an important role to modulate contraction of EUS [2]. Pelvic nerves are the most important pathway for initiating the voiding, the afferent pathway of pelvic nerves consist of small myelinated $(\mathrm{A} \delta)$ and unmyelinated (C) axons and transport impulses from the receptors of bladder wall [3, 4]. However, after spinal cord injury and some neurological disorders, bladder and EUS contractions become uncoordinated and result in bladder and EUS contract simultaneously during voiding, which is termed detrusor sphincter dyssynergia (DSD) and could cause serious medical complications, such as high bladder pressures, urinary tract infections, renal failure, autonomic dysreflexia, and hydronephrosis [5]. Residual urine in the bladder can also lead to lower urinary tract infection.

The conventional treatment of DSD is clean intermittent selfcatheterization (CISC) by using balloon dilation of the EUS or intraurethral stent placement combined with anti-muscarinic drugs. One alternative method to replace CISC is Sphincterotomy, which provides an effective surgical method to lower bladder pressure and increase voiding efficiency. However, the surgical process may have risks of complications, such as permanent incontinence, loss of reflex defecation, and loss of sexual function, for long term DSD patients. There is a severe syndrome during the storage phase of the micturition cycle called detrusor overactivity, serve detrusor overactivity may lead to urgency, frequency and incontinence. The last 5 decades have seen growing importance placed on research in using electrical stimulation (ES) to treat detrusor overactivity [6], many researchers have become involved in this therapy around the world. Andre'E aptly pointed out, using different stimulation parameters on different nerve were an important issue for clinical to treat detrusor overactivity [7]. Our research team recently used pulsed radio-frequency (PRF) electrical stimulation to successfully inhibit $\mathrm{C}$-fiber excitatory responses and obstruct the pain conduction signal, the effect can maintain for 3-6 months [8, 9]. To the best of our knowledge, few studies explored the feasibility of PRF electrical stimulation in the treatment of detrusor overactivity. The purpose of this study was to establish an in vitro experimental setup for the measurement of the effectiveness of low-volt PRF electrical stimulation on pelvic nerve in detrusor overactivity rats. The cystometrograms (CMGs) measurement was utilized to assess the effects of PRF on the pelvic nerve, respectively. This study may be critically important in laying the groundwork for understanding how to use PRF for neuromodulation in detrusor overactivity.

\section{MATERIALS AND METHODS}

All protocols involving the use of animals in this study were approved by the animal care committee at Taipei Medical University and Hospital. Forty-five female Sprague Dawley rats weighing 275-320 g were used. All the rats were anesthetized with urethane $(1.2 \mathrm{~g} / \mathrm{kg})$ and then intravesically infused with $0.5 \%$ acetic acid (AA) to induce detrusor overactivity. They were equally divided into two groups $(\mathrm{n}=$ 15 each) to quantify the effects of PRF neuromodulation. The rats that received AA infusion but not PRF were included in the first group. The rats that received AA infusion and PRF on the pelvic nerve were included in the second group. The urinary bladder was exposed through a midline abdominal incision, and a polyethylene (PE) tube 60 (1.0-mm ID and 1.5$\mathrm{mm}$ OD) was inserted into the bladder lumen for bladder pressure measurements. The bladder end of the PE tube was heated to form a collar and then passed through a small incision at the apex of the bladder dome. The tube was secured using a purse-string suture, and the abdominal wall was closed using nylon sutures. The PE tube was then connected through a three-way stopcock to an infusion pump for filling with 
physiological saline and to a pressure transducer (P23XL-1, Becton Dickinson, NJ, USA) for monitoring bladder pressure. A bipolar cuff electrode was placed on the unilateral pelvic nerve, which connected to the PRF electrical stimulator. The temperature of the rats was maintained at $36^{\circ} \mathrm{C}-38^{\circ} \mathrm{C}$ by using a recirculating water blanket, and the tail vein was catheterized for fluid and drug administration. The bladder pressure was first amplified and sampled using a biological signal acquisition system (Biopac MP 36, BIOPAC Systems, Inc., CA USA). The schematic diagram of the main experimental setup is presented in Fig. 1A. PRF parameters were 80-ms biphasic pulses at $2-\mathrm{Hz}$ frequency, and each pulse consisted of $500-\mathrm{kHz}$ sinusoid radiofrequency (RF) waves; the stimulating intensity was $\pm 3 \mathrm{~V}$ (Fig. 1B). PRF stimulation was delivered on the unilateral pelvic nerve in the rats for a short period of $300 \mathrm{~s}$ in all stimulation trials. The stimulator was $14 \mathrm{~mm} \times 20 \mathrm{~mm} \times$ $4 \mathrm{~mm}$ in size (Fig. 2) and connected to the cuff electrode, the temperature of the electrode was always below $38^{\circ} \mathrm{C}$. All the rats in the two groups underwent urodynamic measurements that usually began $1-2 \mathrm{~h}$ after anesthesia induction. After manual emptying of the bladder, transvesical cystometry was performed at an infusion rate of $0.2 \mathrm{~mL} / \mathrm{min}$ with physiological saline at room temperature. The urethra was opened to enable the elimination of fluid during micturition, and cystometric measurements were recorded in the control group. Subsequently, after a 30-min rest period, the first CMG recording was obtained with $0.5 \% \mathrm{AA}$ infusion to induce detrusor overactivity. These CMGs were recorded without PRF stimulation to measure the $\mathrm{BC}$ of the detrusor overactivity. After a 30-min rest period, PRF electrical currents were applied on the unilateral pelvic nerve in the rats of the PRF intervention groups, which comprised a short period of $300 \mathrm{~s}$ and a PRF waveform of $500 \mathrm{kHz}$. For the rats in the control group, no electrical current output was applied to the target nerves. After a 30-min recovery period, the empty bladder was infused with $0.5 \%$ AA for the second CMG measurement, and the bladder was emptied after the CMG measurement. The third to fifth CMG measurements were then conducted using transvesical AA infusion at every 1-h interval to permit recovery of the bladder reflexes and residual urine collection. The time profile of the protocol is presented in Fig. 1C. The neural pathway that control storage and micturition is shown in Fig 3. Storage reflexes are activated during bladder filling and are organized by the spinal cord. During the storage reflexes, the bladder detrusor innervated by the storage center of pontine through pelvic nerve relaxes to fulfill more urine, and the EUS controlled by the pudendal nerve to keep the resistance of urethral outlet at the same time. The common clinical symptoms of detrusor overactivity patients are losing control of bladder and urinating frequently due to the decrease of bladder capacity. According to the mechanism of PRF electrical stimulation, we suppose that using PRF on pelvic nerve probably block impulses from the mechanoreceptors receptors in the bladder wall, thereby increasing the capacity of bladder. Oppositely, voiding is mediated by reflex mechanisms that are organized in the brain. During the micturition reflexes, the bladder contracts to raise the intravesical pressure (IVP), and
EUS, which controlled by the micturition center of pontine through the pudendal nerve, relaxes to lower the resistance of urethral outlet simultaneously. In addition, there are many reflexes are involved in the act of micturition which affects the coordination between bladder and EUS, particularly the pudendal-pelvic pathway. When urine pass through the urethra, the impulses from the receptor of urethra that pass to the spinobulbospinal pathway and further raise bladder activity. In our hypothesis, PRF electrical stimulation on pudendal nerve could directly affect EUS and pudendal-pelvic pathway, and by doing so improved the lower urinary tract (LUT) function in detrusor overactivity.
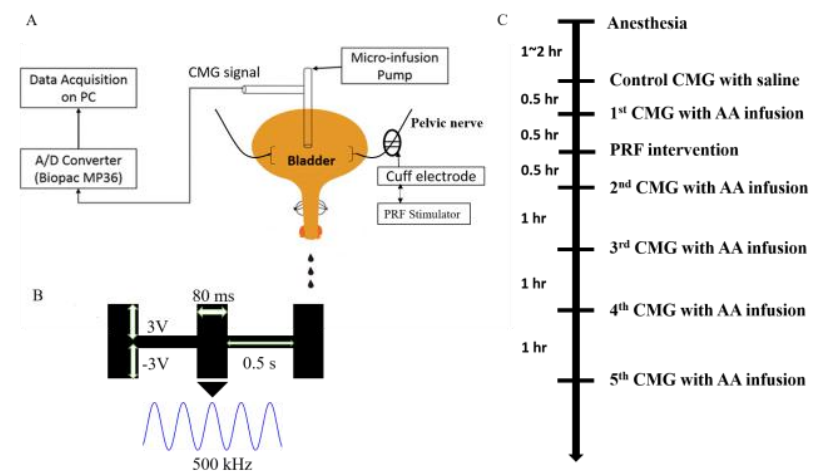

Fig. 1. (A) The measurement system setup was used to record cystometrograms (CMGs) during transvesical saline or acetic acid infusion in the rat. (B) Parameters of the PRF waveform applied on the unilateral pelvic or pudendal nerve. (C) Time profile of experiment protocols for the CMG measurement with saline, the first CMG measurement with acetic acid (AA), PRF stimulation pretreatment, and the second to fifth CMG measurements with AA.

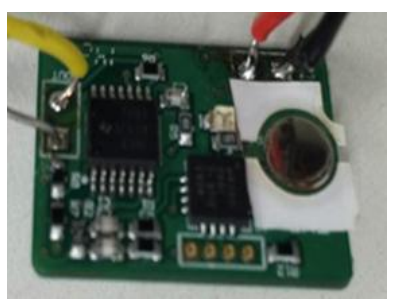

Fig. 2. PRF stimulator was $14 \mathrm{~mm} \times 20 \mathrm{~mm} \times 4 \mathrm{~mm}$ in size

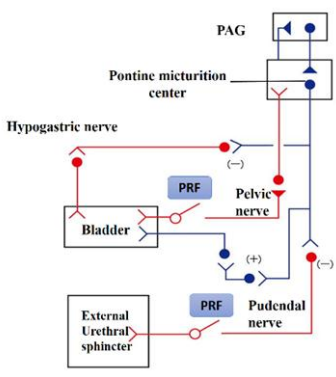

Fig. 3. Neural pathway that control storage and micturition
The researcher analyzing the CMG activity was blinded to the status of the rats, and the data were analyzed by another experienced researcher. The following two cystometric parameters were measured in all rats to quantify the effects of PRF electrical stimulation: $\mathrm{BC}$, defined as the infused volume of saline sufficient to induce the first voiding contraction, exceeding $15 \mathrm{~cm} \mathrm{H} 2 \mathrm{O}$ (the time interval [point $\mathrm{b}^{\prime}$ - point $\mathrm{a}^{\prime}$ ] multiplied by the infusion rate $[0.2 \mathrm{~mL} / \mathrm{min}])$, as depicted in Fig. 4; 


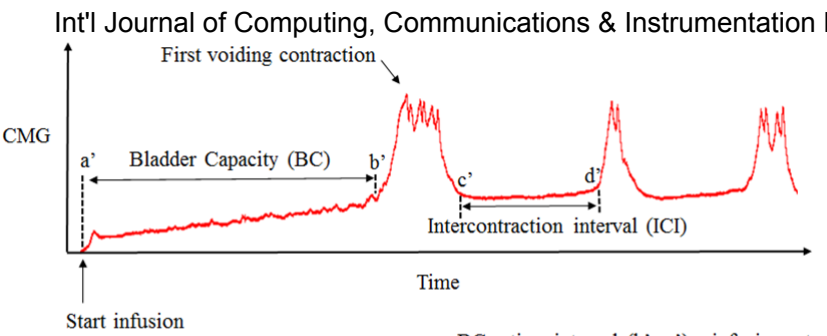

Fig. 4. Typical cystometrography (CMG) measurement at a constant infusion rate of $0.2 \mathrm{~mL} / \mathrm{min}$. CMG parameters include bladder capacity $(\mathrm{BC}$; the time interval [point $\mathrm{b}^{\prime}-$ point $\mathrm{a}^{\prime}$ ] multiplied by the infusion rate $[0.2 \mathrm{~mL} / \mathrm{min}]$ )

\section{RESULTS}

\section{Effects of PRF Stimulation Pretreatment of the Pelvic Nerve}

Fig 5 presents a striking effect of $\mathrm{BC}$ after AA irritation. The $\mathrm{BC}$ was significantly decreased when detrusor overactivity was induced. Compared to the saline control (Fig. 6), the BC of AA group reduced to a mean of $65 \% \pm 7 \%$ of control BC (15 rats) during saline infusion. Then, after 5 min PRF electrical stimulation on pelvic nerve, the CMGs would be measured for 4 fours, and BC would be increased by $107 \% \pm 2 \%, 105 \% \pm$ $3 \%, 110 \% \pm 7 \%$, and $102 \% \pm 2 \%$ in following first, second, third, and fourth hours respectively.

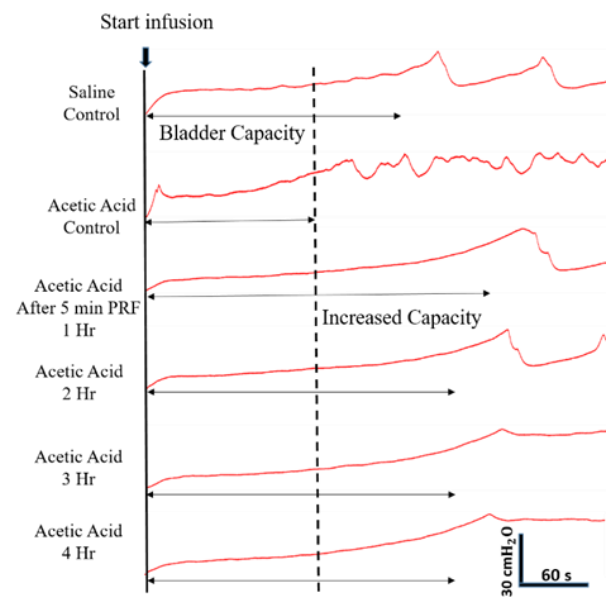

Fig. 5. Effects of pulsed radiofrequency (PRF) stimulation pretreatment of the pelvic nerve on bladder activity with repeated transvesical AA infusion at 1-h intervals. The cvstometric parameters were auantized in the rats $(n=15)$.

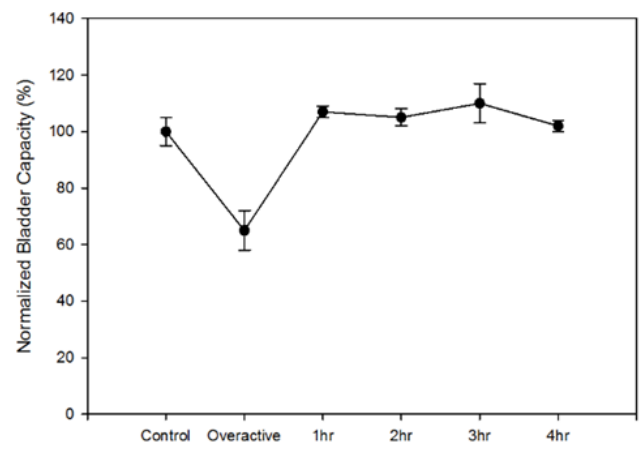

Fig. 6. Comparison of normalized bladder capacity

\section{CONCLUSION}

The present study quantified the effects of PRF electrical stimulation on the pelvic nerve of rats with detrusor overactivity. PRF electrical stimulation of the pelvic nerve significantly suppressed AA-induced detrusor overactivity. In the clinic, the standard therapy for an detrusor overactivity involves a drug regimen of anticholinergics, such as oxybutynin, tolterodine, solifenacin, fesoterodine, and trospium; however, the usefulness of these regimens are limited due to potential side effects, the route of administration, differences in release rates, dosing, and the specificity of cholinergic receptor on which the drugs act. Functional electrical stimulation (FES) circumvents these problems by acting directly on the micturition reflex. Research using ES to treat detrusor overactivity has increased in recent years, while another minimally invasive technique, percutaneous tibial nerve stimulation, is currently being tested on rats with detrusor overactivity. Percutaneous tibial nerve stimulation is more technically difficult to perform resulting in reduced trial numbers and less statistically significant outcomes compared with trials using pelvic floor electrical stimulation. Nevertheless, only a temporary improvement may be achieved in some rats, and recurrence of symptoms is encountered after a few weeks or months [10].

PRF stimulation is widely used to efficiently treat chronic pain, while reducing paraesthesia and other potential side effects; however, the mechanism of its analgesic action is not well understood. The pain pathway is a more complex sensory nerve transmission than simply relaying sensory information from nociceptors to the brain. Although traditional ES treatment for detrusor overactivity does usually restore control, it involves repeat stimulation during voiding and the required frequency of these treatments are demanding, which is not readily acceptable to all patients. According to our results, a 5 min PRF on the pelvic nerve induced a long-lasting inhibition and significantly increased bladder capacity from $65 \%$ to $106 \%$. These results provide alternative PRF conditions $(500 \mathrm{KHz})$ and frequencies $(2.5 \mathrm{~Hz} \sim 10 \mathrm{KHz})$ to achieve the desired inhibition, thus providing valuable information for understanding the clinical application of PRF neuromodulation in the treatment of detrusor overactivity. The present study is just the first step in exploring the effect of PRF electrical stimulation on the lower urinary tract. These findings lead us to believe that PRF neuromodulation on the lower urinary tract shows great potential for treating patients with detrusor overactivity.

\section{ACKNOWLEDGMENT}

This study was supported by grants from the Ministry of Science and Technology (MOST103-2221-E-038-007-MY3 and NSC 102-2320-B-002-040 -MY2) and by the R\&D Foundation of Urological Medicine, Taiwan. 


\section{REFERENCES}

[1] S. J. Shefchyk, "Spinal cord neural organization controlling the urinary bladder and striated sphincter," Elsevier Science, vol. 137, p. 12, 2002.

[2] A. M. Murphy, R. M. Krlin, and H. B. Goldman, "Treatment of overactive bladder: what is on the horizon?," International Urogynecology Journal, vol. 24, pp. 5-13, 2012.

[3] L. Brubaker, "Electrical stimulation in overactive bladder," Urology, vol. 55, pp. 17-23, 5// 2000.

[4] N. Y. Siddiqui, J. M. Wu, and C. L. Amundsen, "Efficacy and adverse events of sacral nerve stimulation for overactive bladder: A systematic review," Neurourology and Urodynamics, vol. 29, pp. S18-S23, 2010.

[5] W. M. White, J. D. Mobley Iii, R. Doggweiler, C. Dobmeyer-Dittrich, and F. A. Klein, "Sacral Nerve Stimulation for Refractory Overactive Bladder in the Elderly Population," The Journal of Urology, vol. 182, pp. $1449-1452,10 / / 2009$.

[6] A. E. Snellings and W. M. Grill, "Effects of stimulation site and stimulation parameters on bladder inhibition by electrical nerve stimulation," BJU international, vol. 110, pp. 136-143, 2012.

[7] C. Tai, B. Shen, J. Wang, H. Liu, J. Subbaroyan, J. R. Roppolo, et al., "Inhibition of bladder overactivity by stimulation of feline pudendal nerve using transdermal amplitude- modulated signal (TAMS)," BJU international, vol. 109, pp. 782-787, 2012.

[8] M. L. Lin, W. T. Lin, R. Y. Huang, T. C. Chen, S. H. Huang, C. H. Chang, et al., "Pulsed radiofrequency inhibited activation of spinal mitogen- activated protein kinases and ameliorated early neuropathic pain in rats," European Journal of Pain, vol. 18, pp. 659-670, 2014.

[9] W. Lin, C. H. Chang, C. Cheng, M. C. Chen, Y. Wen, C. Lin, et al., "Effects of low amplitude pulsed radiofrequency stimulation with different waveform in rats for neuropathic pain," in Engineering in Medicine and Biology Society (EMBC), 2013 35th Annual International Conference of the IEEE, 2013, pp. 3590-3593.

[10] Y. Matsuta, J. R. Roppolo, W. C. de Groat, and C. Tai, "Poststimulation inhibition of the micturition reflex induced by tibial nerve stimulation in rats," Physiological reports, vol. 2, 2014. 